PROCEEDINGS OF THE

AMERICAN MATHEMATICAL SOCIETY

Volume 135, Number 11, November 2007, Pages 3485-3493

S 0002-9939(07)08906-X

Article electronically published on June 29, 2007

\title{
ON FINITELY INJECTIVE MODULES AND LOCALLY PURE-INJECTIVE MODULES OVER PRÜFER DOMAINS
}

\author{
LUIGI SALCE
}

(Communicated by Bernd Ulrich)

\begin{abstract}
Over Matlis valuation domains there exist finitely injective modules which are not direct sums of injective modules, as well as complete locally pure-injective modules which are not the completion of a direct sum of pureinjective modules. Over Prüfer domains which are either almost maximal, or $h$-local Matlis, finitely injective torsion modules and complete torsion-free locally pure-injective modules correspond to each other under the Matlis equivalence. Almost maximal Prüfer domains are characterized by the property that every torsion-free complete module is locally pure-injective. It is derived that semi-Dedekind domains are Dedekind.
\end{abstract}

\section{INTRODUCTION}

A result in the Ph.D. thesis of $\mathrm{H}$. Bass, whose proof can be found in [3], states that a ring is left Noetherian if and only if the class of the injective modules is closed under taking direct sums. Ramamurthi and Rangaswamy [15] noted that left Noetherian rings are also characterized by the stronger condition that finitely injective modules are injective; recall that a module $M$ is finitely injective if every finite set of elements of $M$ is contained in an injective submodule; obviously, direct sums of injective modules are "trivial" examples of finitely injective modules.

Thus the following question naturally arises: if $R$ is a non-Noetherian ring, is the class of the direct sums of injective modules strictly contained in the class of finitely injective modules? In other words, does a finitely injective $R$-module exist which fails to be a direct sum of injective modules? Note that such a module must be at least $\aleph_{1}$-generated, since a countably generated finitely injective module is necessarily a direct sum of injectives (see Proposition 1.1).

The first goal of this paper is to show that the answer to the above question is positive when $R$ is a non-Noetherian Matlis valuation domain (recall that a domain is Matlis if the projective dimension of the field of quotients $Q$ of $R$ is $\leq 1$ ).

The tool to obtain the required finitely injective module is a construction that was first performed by Hill [1] to exhibit $\aleph_{1}$-separable $p$-groups which are not direct sums of countable groups. Later on, this construction was modified by P. A. Griffith [9] to obtain flat $\aleph_{1}$-separable torsionless modules over non-left perfect rings,

Received by the editors February 6, 2006 and, in revised form, August 21, 2006.

2000 Mathematics Subject Classification. Primary 13A05; Secondary 13C11, 13F05.

Key words and phrases. Finitely injective modules, locally pure-injective modules, Matlis equivalence.

The research of this author was supported by MIUR, PRIN 2005.

(C)2007 American Mathematical Society Reverts to public domain 28 years from publication 
which fail to be direct sums of countably generated modules (here $\aleph_{1}$-separable means that each countable subset belongs to a countably generated direct summand). B. Zimmermann-Huisgen [20] generalized Hill's and Griffith's constructions (and similar ones by Gruson-Jensen [10] and W. Zimmermann [18]) to prove that all $\aleph_{1}$-separable modules over an arbitrary associative unital ring $R$ are trivial (i.e., direct sums of countably generated modules) if and only if $R$ has pure global dimension zero, namely, every pure inclusion of left $R$-modules splits.

We will adapt the construction in 20] to the setting of divisible modules over a non-Noetherian almost maximal Matlis valuation domain $R$, obtaining $\aleph_{1}$-generated finitely injective torsion modules whose only injective summands are isomorphic to finite direct sums of $Q / R$. Recall that over such a domain $R$ all divisible modules are finitely injective (see [6] and [16]). Then we will extend the above result eliminating the almost maximal assumption.

Using the Matlis equivalence between $h$-divisible torsion modules and torsionfree complete modules, we obtain an example of a complete locally pure-injective torsion-free module over a non-Noetherian Matlis valuation domain, which fails to be the completion of a direct sum of pure-injective modules (see [19] for a study of locally pure-injective modules). Furthermore, we characterize almost maximal Prüfer domains $R$ by means of the property that all torsion-free complete $R$-modules are locally pure-injective. As a subproduct of this result we derive that the semiDedekind domains, introduced by S. B. Lee in [13, are in fact Dedekind domains.

The latter goal of the paper is to show that, over Prüfer domains which are either almost maximal, or $h$-local Matlis, finitely injective torsion modules correspond, under the Matlis equivalence, to complete torsion-free locally pure-injectve modules.

\section{NON-TRIVIAL Finitely inJECTIVE MODULES}

It is useful to introduce some definitions. B. Zimmermann-Huisgen [20] calls a module over an arbitrary ring $\aleph_{1}$-separable if each countable subset belongs to a countably generated direct summand (this notion differs from that defined in 4 , p. 87]); she also calls an $\aleph_{1}$-separable module "trivial" if it is a direct sum of countably generated submodules. Inspired by this definition, we say that a finitely injective module is trivial if it is a direct sum of injective modules. Given an infinite cardinal $\kappa$, a module $D$ is said to be $\kappa-\oplus$ injective if every subset of $D$ of cardinality $<\kappa$ is contained in a submodule $D^{\prime}$ of $D$ which is a direct sum of injective modules. If, furthermore, $D^{\prime}$ can be chosen being a summand of $D$, then $D$ is said to be separable $\kappa-\oplus$ injective.

A finitely injective module $D$ is trivial exactly if it is $\kappa$ - $\oplus$ injective for all $\kappa$. Note that a separable $\aleph_{0}-\oplus$ injective module is nothing else than a finitely injective module. The next result is similar to Proposition 2.5 in [19] (which deals with locally pure injective modules).

Proposition 1.1. A finitely injective module is $\aleph_{1}-\oplus$ injective.

Proof. Let $M$ be a finitely injective module and $\left(x_{n}\right)_{n \in \omega}$ a sequence of elements in $M$. We argue by induction. Let $E_{1} \oplus \cdots \oplus E_{n} \oplus N=M$, where $E_{1} \oplus \cdots \oplus E_{n}$ is a finite direct sum of injective submodules of $M$ containing $x_{1}, \ldots, x_{n}$. Let $x_{n+1}=y+z$, with $y \in E_{1} \oplus \cdots \oplus E_{n}$ and $z \in N$. Since $N$ is finitely injective, $z$ belongs to an injective submodule $E_{n+1}$ of $N$, thus $E_{1} \oplus \cdots \oplus E_{n+1}$ contains $x_{1}, \ldots$, $x_{n+1}$. There follows that $\bigoplus_{n \in \omega} E_{n}$ contains $\left(x_{n}\right)_{n \in \omega}$. 
An immediate consequence of Proposition 1.1] is that countably generated finitely injective modules are trivial, i.e., direct sums of injective modules.

Most of this section is devoted to proving the following.

Theorem 1.2. Let $R$ be a non-Noetherian Matlis almost maximal valuation domain. Then there exists a non-trivial separable $\aleph_{1}-\oplus$ injective module $D$.

Proof. Let $0<R r_{1}<R r_{2}<\cdots<R r_{n}<\cdots$ be a strictly increasing sequence of principal ideals of $R$, and let $J=\bigcup_{n \in \omega} R r_{n}$. For each ordinal $\sigma \leq \aleph_{1}$ set

$$
S_{\sigma}=\bigoplus_{\rho<\sigma} K_{\rho}, \quad P_{\sigma}=\prod_{\rho<\sigma} K_{\rho}
$$

where $K_{\rho}=K=Q / R$ for all $\rho$.

First we construct a countably generated submodule $T_{\omega}$ of $P_{\omega}$ containing $S_{\omega}$, which is isomorphic to $\bigoplus_{\aleph_{0}} K$ such that $S_{\omega}$ is not a direct summand of $T_{\omega}$.

Let $\mathbf{x}=\left(x_{n}\right)_{n \in \omega} \in P_{\omega}$ be the element defined by setting, for each $n \in \omega$, $x_{n}=r_{n}^{-1}+R$. Then $\operatorname{Ann}_{R}(\mathbf{x})=R r_{1}$, hence $R \mathbf{x} \cong R / R r_{1}$, and an easy computation shows that $A_{n}\left(\mathbf{x}+S_{\omega}\right)=J$. Let us set

$$
T_{\omega}=S_{\omega}+E_{\omega}
$$

where $E_{\omega}$ denotes the injective envelope of the cyclic module $R \mathbf{x}$ inside the injective module $P_{\omega}$. $E_{\omega}$ is countably generated, since $E_{\omega}=E(R \mathbf{x}) \cong Q / R r_{1} \cong$ $K$. Therefore, $T_{\omega}$ is countably generated. Moreover, since $E_{\omega}$ is uniserial and $\operatorname{Ann}_{R}\left(\mathbf{x}+S_{\omega}\right)=J$, we have

$$
T_{\omega} / S_{\omega} \cong E_{\omega} /\left(E_{\omega} \cap S_{\omega}\right)=E(R \mathbf{x}) / J \mathbf{x} \cong Q / J .
$$

It follows that p.d. $T_{\omega} / S_{\omega}=2$ (see [8, VI, Exercise 3.3]; as usual, p.d. $M$ denotes the projective dimension of the module $M$ ). On the other hand, p.d. $T_{\omega}=1$; in fact, every finitely generated submodule of $T_{\omega}$ is contained in a submodule of the form $K_{1} \oplus \cdots \oplus K_{n}+E_{\omega}$, which is isomorphic to $K_{1} \oplus \cdots \oplus K_{n} \oplus K$, since $\operatorname{Ann}_{R}\left(\mathbf{x}+K_{1} \oplus \cdots \oplus K_{n}\right)=R r_{n+1}$. There it follows that $T_{\omega}$ is coherent, hence is isomorphic to $\bigoplus_{\aleph_{0}} K$. (N.B.: It is easily seen that the module $T_{\omega}$ is isomorphic to the module $T$ constructed in [1, Lemma 3.4]. However, it is important to construct $T_{\omega}$ as above, in order to adapt Griffith's technique.)

Let us now fix a ladder system on the set $\lim \left(\aleph_{1}\right)$ of the limit ordinals in $\aleph_{1}$ (see [4, p. 40]); that is, for each limit ordinal $\lambda<\aleph_{1}$, we fix an increasing sequence $J(\lambda)$ of ordinals: $\sigma_{1}<\sigma_{2}<\cdots<\sigma_{n}<\cdots<\lambda$, whose supremum is $\lambda$. We denote by $S_{J(\lambda)}$ and $P_{J(\lambda)}$, respectively, the two modules $\bigoplus_{\rho \in J(\lambda)} K_{\rho}$ and $\prod_{\rho \in J(\lambda)} K_{\rho}$.

Let us define an element $\mathbf{x}^{\lambda}=\left(x_{\sigma}^{\lambda}\right)_{\sigma<\lambda} \in P_{\lambda}$ in the following way:

$$
x_{\sigma}^{\lambda}= \begin{cases}r_{n}^{-1}+R & \text { if } \sigma=\sigma_{n} \text { for some } n \\ 0 & \text { otherwise }\end{cases}
$$

Let us set

$$
T_{\lambda}=S_{J(\lambda)}+E_{\lambda},
$$

where $E_{\lambda}$ denotes the injective envelope of the cyclic module $R \mathrm{x}^{\lambda}$ inside the injective module $P_{J(\lambda)}$ (we look at $\mathrm{x}^{\lambda}$ as an element of $P_{J(\lambda)}$ in the obvious way). The module $T_{\lambda}$ is a "clone" (B. Zimmermann-Huisgen calls the similar module in her construction a "carbon copy" [20]) of the module $T_{\omega}$ defined above. In particular, $T_{\lambda} / S_{J(\lambda)} \cong Q / J ;$ hence, p.d. $T_{\lambda} / S_{J(\lambda)}=2$. 
We are now in a position to define the desired finitely injective module $D$. Let us set

$$
D=S_{\aleph_{1}}+\sum_{\lambda \in \lim \left(\aleph_{1}\right)} E_{\lambda}
$$

We will prove that $D$ is separable $\aleph_{1}-\oplus$ injective and is not a direct sum of injective modules. Actually, we will prove more: the only injective summands of $D$ are isomorphic to $(Q / R)^{n}(n \in \mathbb{N})$. Note that $D$ is the union of an increasing chain of submodules: $D=\bigcup_{\sigma<\aleph_{1}} D_{\sigma}$, where, for each $\sigma<\aleph_{1}$,

$$
D_{\sigma}=S_{\sigma}+\sum_{\lambda \in \lim (\sigma)} E_{\lambda} .
$$

Each module $D_{\sigma}$ is divisible, countably generated and coherent; hence, $D_{\sigma} \cong$ $\bigoplus_{\aleph_{0}} K$ for all $\sigma<\aleph_{1}$. We need the following technical lemmas.

Lemma 1.3. If $\lambda \in \lim \left(\aleph_{1}\right)$, then $D \cap P_{\lambda}=D_{\lambda}+E_{\lambda}$.

Proof. The inclusion $D \cap P_{\lambda} \geq D_{\lambda}+E_{\lambda}$ is obvious. Conversely, let $x=\sum_{1 \leq i \leq n} k_{\rho_{i}}+$ $\sum_{1 \leq j \leq m} e_{\lambda_{j}} \in D \cap P_{\lambda}$, where $k_{\rho_{i}} \in K_{\rho_{i}}, e_{\lambda_{j}} \in E_{\lambda_{j}}$, and $\lambda_{1}<\cdots<\lambda_{m}$ are limit ordinals. Since $J\left(\lambda_{m}\right)$ has finite intersection with $J\left(\lambda_{j}\right)$ for each $j, x \in P_{\lambda}$ implies that $\lambda_{m} \leq \lambda$. Then $\sum_{1 \leq j \leq m} e_{\lambda_{j}} \in P_{\lambda}$ and consequently also $\sum_{1 \leq i \leq n} k_{\rho_{i}} \in P_{\lambda}$. Therefore, $\rho_{i}<\lambda$ for all $i$; hence, $\sum_{1 \leq i \leq n} k_{\rho_{i}}+\sum_{1 \leq j \leq m-1} e_{\lambda_{j}} \in D_{\lambda}$ and $e_{\lambda_{m}} \in E_{\lambda}$, so that $x \in D_{\lambda}+E_{\lambda}$ as desired.

Lemma 1.4. If $\lambda \in \lim \left(\aleph_{1}\right)$, then

(1) $D_{\lambda}=\left(D_{\lambda} \cap \prod_{\rho \in \lambda \backslash J(\lambda)} K_{\rho}\right) \oplus S_{J(\lambda)}$;

(2) $D_{\lambda+1}=\left(D_{\lambda} \cap \prod_{\rho \in \lambda \backslash J(\lambda)} K_{\rho}\right) \oplus T_{\lambda} \oplus K_{\lambda}$;

(3) $D_{\lambda+1}=D \cap \prod_{\rho \leq \lambda} K_{\rho}$.

Proof. (1) Using the notation in the proof of Lemma 1.3. let $x=\sum_{1<i \leq n} k_{\rho_{i}}+$ $\sum_{1 \leq j \leq m} e_{\lambda_{j}} \in D_{\lambda}$, where $\rho_{1}<\cdots<\rho_{m}<\lambda$, and $\lambda_{1}<\cdots<\lambda_{m}<\lambda$ are limit ordinals. Since $J(\lambda)$ has finite intersection with $J\left(\lambda_{j}\right)$ for each $j$, each $e_{\lambda_{j}}$ has only finitely many components in $S_{J(\lambda)}$. Let $y$ denote the element of $S_{J(\lambda)}$ which is the sum of all of these components for $1 \leq j \leq m$, and of all the elements $k_{\rho_{i}}$ such that $\rho_{i} \in J(\lambda)$. Then $x-y \in D_{\lambda} \cap \prod_{\rho \in \lambda \backslash J(\lambda)} K_{\rho}$, so we are done.

(2) $D_{\lambda+1}=\left(D_{\lambda}+E_{\lambda}\right) \oplus K_{\lambda}=\left(\left(\left(D_{\lambda} \cap \prod_{\rho \in \lambda \backslash J(\lambda)} K_{\rho}\right) \oplus S_{J(\lambda)}\right)+E_{\lambda}\right) \oplus K_{\lambda}=$ $\left(D_{\lambda} \cap \prod_{\rho \in \lambda \backslash J(\lambda)} K_{\rho}\right) \oplus T_{\lambda} \oplus K_{\lambda}$.

(3) Using Lemma 1.3 and the equality $P_{\lambda+1}=P_{\lambda} \oplus K_{\lambda}$, we get $D_{\lambda+1} \leq D \cap$ $\left(P_{\lambda} \oplus K_{\lambda}\right)=\left(D \cap P_{\lambda}\right) \oplus K_{\lambda}=\left(D_{\lambda}+E_{\lambda}\right) \oplus K_{\lambda} \leq D_{\lambda+1}$; hence, $D_{\lambda+1}=D \cap$ $\left(P_{\lambda} \oplus K_{\lambda}\right)=D \cap \prod_{\rho \leq \lambda} K_{\rho}$

Lemma 1.5. If $\lambda \in \lim \left(\aleph_{1}\right)$, then

$$
D=D_{\lambda+1} \oplus\left(D \cap \prod_{\lambda<\rho<\aleph_{1}} K_{\rho}\right) .
$$

Proof. By Lemma 1.4 (3), we must prove that $D=\left(D \cap \prod_{\rho \leq \lambda} K_{\rho}\right) \oplus(D \cap$ $\left.\prod_{\lambda<\rho<\aleph_{1}} K_{\rho}\right)$. Let $x=\sum_{1 \leq i \leq n} k_{\rho_{i}}+\sum_{1 \leq j \leq m} e_{\lambda_{j}} \in D$, where $\rho_{1}<\cdots<\rho_{k} \leq \lambda<$ $\rho_{k+1}<\cdots<\rho_{m}$, and $\lambda_{1}<\cdots<\lambda_{h} \leq \lambda<\lambda_{h+1}<\cdots<\lambda_{m}$ are limit ordinals. 
Let $y$ denote the element of $\prod_{\rho \leq \lambda} K_{\rho}$ which is the sum of the finitely many components in $\sum_{\rho \leq \lambda} K_{\rho}$ of the elements $e_{\lambda_{j}}$ for $h+1 \leq j \leq m$. Then

$$
x=\left(\sum_{1 \leq i \leq k} k_{\rho_{i}}+\sum_{1 \leq j \leq h} e_{\lambda_{j}}+y\right)+\left(\sum_{k<i \leq n} k_{\rho_{i}}+\sum_{h<j \leq m} e_{\lambda_{j}}-y\right),
$$

where the first summand belongs to $D \cap \prod_{\rho \leq \lambda} K_{\rho}$, and the second summand belongs to $D \cap \prod_{\lambda<\rho<\aleph_{1}} K_{\rho}$. Consequently, $D \subseteq D_{\lambda+1} \oplus\left(D \cap \prod_{\lambda<\rho<\aleph_{1}} K_{\rho}\right)$. The converse inclusion is trivial.

We now continue the proof of Theorem 1.2, By Lemma 1.5, $D_{\lambda+1}$ is a direct summand of $D$ for each limit ordinal $\lambda<\aleph_{1}$; there it follows that $D$ is not an injective module, as $D_{\lambda+1} \cong \bigoplus_{\aleph_{0}} K$ is not injective. $D$ is separable $\aleph_{1}-\oplus$ injective, since a countable subset of $D$ is contained in $D_{\lambda+1}$ for some limit ordinal $\lambda<\aleph_{1}$, and $D_{\lambda+1}$ is a direct sum of injective modules which is a summand in $D$. Finally, we must show that $D$ is not a direct sum of injective modules. First we observe that $D$ is not isomorphic to $\bigoplus_{\aleph_{1}} K$; in fact, from Lemma 1.4 we derive that $D_{\lambda+1} / D_{\lambda} \cong$ $\left(T_{\lambda} / S_{J(\lambda)}\right) \oplus K$; hence, p.d. $D_{\lambda+1} / D_{\lambda}=2$ for all limit ordinals $\lambda$; a well-known result by Eklof (see [7, p. 75]) implies that p.d. $D=2$, while p.d. $\bigoplus_{\aleph_{1}} K=1$.

It remains to show that $D$ is not a direct sum of injective modules of some different kind. Assume, by way of contradiction, that $D$ has a direct summand $E$ which is the injective hull of a module $B \cong \bigoplus_{\aleph_{0}} K$. Then there exists a countable ordinal $\lambda$ such that $B \leq D_{\lambda+1}$. As $B$ is essential in $E$, we get from Lemma 1.5 that $E \cap\left(D \cap \prod_{\lambda<\rho<\aleph_{1}} K_{\rho}\right)=0$. This implies that $E$ embeds as a direct summand in $D_{\lambda+1}$, which is impossible, since $E$ is not countably generated.

We now extend Theorem 1.2 to arbitrary non-Noetherian Matlis valuation domains. We need the following technical lemma; recall that, if $S$ denotes a maximal immediate extension of $R$, then $E(Q / R) \cong Q S / S$ (see [5]), which is an injective $S$-module, and, as $S$ is a flat overring of $R$, injective $S$-modules are also injective as $R$-modules (see [12, p. 62, 3.6A] or [8, IX, Exercise 1.3]).

Lemma 1.6. Let $S$ be a maximal immediate extension of the valuation domain $R$, and let $E$ be the injective hull as an $R$-module of a module of the form $B=$ $\bigoplus_{\aleph} Q S / S$ for some $\aleph$. Then $E$ is an injective $S$-module.

Proof. Let $A$ be the injective hull of $B$ as an $S$-module. Then $A$ is an injective $R$ module which decomposes as an $R$-module in the form $A=E \oplus E^{\prime}$. If $E^{\prime} \neq 0$, then $E^{\prime}$ contains a direct $R$-summand $C \cong Q S / S$. But this is impossible, since $B \oplus C$ is also a direct decomposition of $S$-modules, while $B$ is an essential $S$-submodule of $A$.

Theorem 1.7. Let $R$ be a non-Noetherian Matlis valuation domain. Then there exists a non-trivial finitely injective module $D$.

Proof. Let $S$ be a maximal immediate extension of $R$. Let $D$ be a finitely injective $S$-module constructed as in Theorem 1.2 with $S$ instead of $R$; this makes sense, since $S$ is a non-Noetherian Matlis maximal valuation domain. Clearly finitely injective $S$-modules are also finitely injective as $R$-modules; hence, it is enough to show that $D$ is not a direct sum of injective $R$-modules. We will show more: the only injective summands of $D$ as an $R$-module are isomorphic to finite direct sums 
of copies of $E(Q / R)$. Let us assume, by way of contradiction, that $D$ decomposes as an $R$-module in the following way:

$$
D=E\left(\bigoplus_{\aleph_{0}} Q / R\right) \oplus D^{\prime} \cong E\left(\bigoplus_{\aleph_{0}} Q S / S\right) \oplus D^{\prime}
$$

(note that an indecomposable injective $R$-summand of $D$ is necessarily isomorphic to $E(Q / R)$, since $D$ is a coherent $R$-module). To reach the contradiction it is enough to prove that $E\left(\bigoplus_{\aleph_{0}} Q S / S\right)$ is the injective hull of $\bigoplus_{\aleph_{0}} Q S / S$ as an $S$-module, and this is ensured by Lemma 1.6.

\section{Finitely injective modules and Matlis equivalence}

A classical result by Matlis establishes an equivalence between the category of $h$-divisible torsion modules over an arbitrary domain $R$ on one side, and the category of torsion-free complete $R$-modules on the other side; it is understood that the completion is made with respect to the $R$-topology. The two inverse correspondences send the $h$-divisible torsion $R$-module $D$ to the torsion-free complete $R$-module $\operatorname{Hom}_{R}(K, D)(K=Q / R)$, and the torsion-free complete $R$-module $M$ to the $h$-divisible torsion $R$-module $K \otimes_{R} M$ (see [8, VIII.2]).

As finitely injective torsion modules are $h$-divisible (see [16, Corollary 1.3]), it is natural to ask which torsion-free complete modules correspond to them in the Matlis equivalence. Recall that, by a Warfield result [17] (see [8, VIII.2.9]), injective torsion modules and torsion-free Warfield cotorsion modules correspond to each other in the Matlis equivalence.

We will investigate the above question over Prüfer domains. Note that, over such a domain $R$, as torsion-free $R$-modules are flat, $R D$-injective and pure-injective modules coincide, as well as Enochs cotorsion and Warfield cotorsion modules, and torsion-free cotorsion modules are pure-injective (see [8, XIII]). Notice also that, in view of the Warfield's result quoted above, it is quite obvious that direct sums of injective torsion $R$-modules and completions of direct sums of torsion-free pureinjective $R$-modules correspond to each other under the Matlis equivalence.

Therefore, natural candidates as modules corresponding to the finitely injective torsion $R$-modules are the complete $l$-pure-injective modules. Recall that a module $M$ over an arbitrary ring is said to be locally pure-injective (l-pure-injective for short), if every element $x \in M$ belongs to a pure-injective summand of $M$. These modules have been investigated recently by W. Zimmermann [19].

The first simple result is the following

Proposition 2.1. Let $M$ be a torsion-free (complete) l-pure-injective module over a domain $R$. Then $M \otimes_{R} K$ is finitely injective.

Proof. It is enough to prove that every element of the form $m \otimes k(0 \neq m \in M, k \in$ $K)$ belongs to an injective summand of $M \otimes_{R} K$. By hypothesis, $M=X \oplus Y$, where $X$ is pure-injective and contains $m$. Then $M \otimes_{R} K=\left(X \otimes_{R} K\right) \oplus\left(Y \otimes_{R} K\right)$, where $X \otimes_{R} K$ contains $m \otimes k$ and is injective, by the Warfield result quoted above.

We give a simple direct proof of the converse of Proposition 2.1 for almost maximal Prüfer domains. Recall that, by [16, every $h$-divisible module over such a domain is finitely injective.

Proposition 2.2. Let $R$ be an almost maximal Prüfer domain, and let $D$ be a torsion h-divisible $R$-module. Then $\operatorname{Hom}_{R}(K, D)$ is l-pure-injective. 
Proof. Let $0 \neq \phi: K \rightarrow D$ be a homomorphism. Since all quotients of $Q$ are injective [2] (see also [8, IX.4.5]), we get that $D=\phi(K) \oplus A$, with $\phi(K)$ injective. Therefore, $\operatorname{Hom}_{R}(K, D)=\operatorname{Hom}_{R}(K, \phi(K)) \oplus \operatorname{Hom}_{R}(K, A)$, where $\operatorname{Hom}_{R}(K, \phi(K))$ is pure-injective by the Warfield's result quoted above, and clearly

$$
\phi \in \operatorname{Hom}_{R}(K, \phi(K)) .
$$

There is another very good reason why the statement of Proposition 2.2 is true: every complete torsion-free module over an almost maximal Prüfer domain is $l$ pure-injective. Actually, with the help of Theorem 2.4 in [16], we can prove the following.

Theorem 2.3. All torsion-free complete modules over a domain $R$ are l-pureinjective if and only if $R$ is an almost maximal Prüfer domain.

Proof. The sufficiency is an obvious consequence of the following facts:

(i) As almost maximal Prüfer domains $R$ are $h$-local, a torsion-free complete $R$ module $M$ (i.e., reduced weakly cotorsion) is isomorphic to $\prod_{P} M^{P}$, where $P$ ranges over $\operatorname{Max}(R)$ and $M^{P}=\operatorname{Hom}_{R}\left(\tilde{R}_{P}, M\right)$ is a complete module over the completion $\tilde{R}_{P}$ of the localization $R_{P}$ (see [14, Corollary 8.6]);

(ii) $\tilde{R}_{P}$ is a maximal valuation domain, and every torsion-free module over such a domain is $l$-pure-injective both as an $\tilde{R}_{P}$-module and as an $R$-module (see 8 , XIV.3]);

(iii) the class of $l$-pure-injective modules is closed under direct products 18 , Prop. 2.4 (2)].

We will prove the necessity by showing that every $h$-divisible torsion $R$-module is finitely injective and then applying Theorem 2.4 in [16. Let $D$ be a torsion $h$-divisible $R$-module. Then $\operatorname{Hom}_{R}(K, D)$ is l-pure-injective, by hypothesis; hence, $D \cong K \otimes_{R} \operatorname{Hom}_{R}(K, D)$ is finitely injective, by Proposition 2.1.

An immediate consequence is the following.

Corollary 2.4. Let $R$ be an almost maximal Prüfer domain. Then the two classes of torsion finitely injective $R$-modules and of torsion-free complete l-pure-injective $R$-modules correspond to each other under the Matlis equivalence.

Another interesting consequence is that semi-Dedekind domains, introduced and investigated by S. B. Lee in [13, are Dedekind. Recall that a domain $R$ is semiDedekind if all $h$-divisible $R$-modules are pure-injective. Lee proved that, if $R$ is semi-Dedekind, then every torsion-free complete $R$-module is pure-injective. As pure-injective modules are trivially $l$-pure-injective, and since semi-Dedekind Prüfer domains are necessarily Dedekind domains, from Theorem 2.3 we derive the following.

Corollary 2.5. A semi-Dedekind domain is a Dedekind domain.

Recall that an almost maximal Prüfer domain is necessarily $h$-local. In the remaining part of this paper we will extend the result in Corollary 2.4 to $h$-local Prüfer domains, but with the additional assumption that they are Matlis.

We will need the next technical result.

Lemma 2.6. Let $R$ be a valuation domain and $\left\{M_{i}\right\}_{i \in I}$ a family of pure-injective torsion-free $R$-modules. Then the completion of $\bigoplus_{i \in I} M_{i}$ in the $R$-topology is $l$ pure-injective. 
Proof. Let $M=\widetilde{\bigoplus_{i \in I} M_{i}}$ be the completion of $\bigoplus_{i \in I} M_{i}$ and let $S$ be a maximal immediate extension of $R$. An element $x \in M$ is the limit of a Cauchy net $\left\{x_{r}\right\}_{0 \neq r \in R}$ of elements in $\bigoplus_{i \in I} M_{i}$, which is an $S$-module. If $\alpha \in S$, then $\alpha x$ is the limit of the net $\left\{\alpha x_{r}\right\}_{0 \neq r \in R}$, hence $\alpha x \in M$; therefore, $M$ is an $S$-module too. Pick a non-zero element $m \in M$; if $x J$ is the pure $R$-submodule of $M$ generated by $x(J \leq Q)$, then $x J S$ is a pure-injective summand of $M$ containing $x$; hence, $M$ is $l$-pure-injective.

We can now prove the converse of Proposition 2.1 for modules over Matlis valuation domains.

Proposition 2.7. Let $R$ be a Matlis valuation domain, and let $D$ be a finitely injective torsion $R$-module. Then $\operatorname{Hom}_{R}(K, D)$ is l-pure-injective.

Proof. Let $0 \neq \phi \in \operatorname{Hom}_{R}(K, D)$. Since $K$ is $\aleph_{0}$-generated, by Proposition 1.1] we have that $\phi(K) \leq \bigoplus_{n \in \omega} E_{n}$, where the $E_{n}$ are injective modules. From the exact sequence

$$
0 \rightarrow \bigoplus_{n \in \omega} E_{n} \rightarrow D \rightarrow X \rightarrow 0
$$

where $X=D / \bigoplus_{n \in \omega} E_{n}$, we obtain the exact sequence

$$
0 \rightarrow \operatorname{Hom}_{R}\left(K, \bigoplus_{n \in \omega} E_{n}\right) \rightarrow \operatorname{Hom}_{R}(K, D) \rightarrow \operatorname{Hom}_{R}(K, X) \rightarrow \operatorname{Ext}_{R}^{1}\left(K, \bigoplus_{n \in \omega} E_{n}\right)
$$

where the last term vanishes, since p.d. $K=1$ and $\bigoplus_{n \in \omega} E_{n}$ is $h$-divisible (see 7 , VII.2.5]). Now $\operatorname{Hom}_{R}\left(K, \bigoplus_{n \in \omega} E_{n}\right)$ is the completion of a direct sum of torsion-free pure-injective modules by the Warfield result in [17]; hence, it is $l$-pure-injective by Lemma 2.6. Furthermore, it is pure in $\operatorname{Hom}_{R}(K, D)$, since $\operatorname{Hom}_{R}(K, X)$ is torsionfree. Clearly $\phi \in \operatorname{Hom}_{R}\left(K, \bigoplus_{n \in \omega} E_{n}\right)$, so it belongs to a pure-injective summand of it, which is as well a direct summand of $\operatorname{Hom}_{R}(K, D)$, being pure in it. Therefore, we can conclude that $\operatorname{Hom}_{R}(K, D)$ is l-pure-injective.

From Theorem 1.7 and Proposition 2.7 we immediately obtain the following.

Corollary 2.8. Let $R$ be a Matlis non-Noetherian valuation domain. There exists a complete l-pure-injective torsion-free $R$-module which fails to be the completion of a direct sum of pure-injective modules.

We can also extend Corollary 2.4 to $h$-local Matlis Prüfer domains.

Corollary 2.9. Let $R$ be an h-local Matlis Prüfer domain. Then the two classes of torsion finitely injective $R$-modules and of torsion-free complete l-pure-injective $R$-modules correspond to each other under the Matlis equivalence.

Proof. By $h$-locality, we can reduce to the local case, namely, to Matlis valuation domains, so the proof follows from Propositions 2.1 and 2.7.

We close the paper with two open questions.

Question 1. Given an arbitrary non-Noetherian ring $R$, does a non-trivial finitely injective $R$-module exist?

Question 2. Given an arbitrary integral domain $R$, which torsion-free complete $R$-modules correspond to the torsion finitely injective $R$-modules under the Matlis equivalence, and which torsion finitely injective $R$-modules correspond to the complete torsion-free $l$-pure-injective $R$-modules? 


\section{REFERENCES}

[1] S. Bazzoni and L. Salce, An independence result on cotorsion theories over valuation domains, J. Algebra 243 (2001), 294-320. MR.1851665 (2002g:13029)

[2] W. Brandal, Almost maximal integral domains and finitely generated modules, Trans. Amer. Math. Soc. 206 (1973), 203-223. MR0325609 (48:3956)

[3] S. U. Chase, Direct products of modules, Trans. Amer. Math. Soc. 97 (1960), 457-473. MR.0120260 (22:11017)

[4] P. Eklof and A. Mekler, Almost free modules, revised edition, North Holland, Elsevier, Amsterdam, 2002. MR 1914985 (2003e:20002)

[5] A. Facchini, Torsion-free covers and pure-injective envelopes over valuation domains, Israel J. Math. 52 (1985), 129-139. MR815608 (87a:13013)

[6] A. Facchini, Absolutely pure modules and locally injective modules, Commutative ring theory (Fès, 1992), Lecture Notes in Pure and Appl. Math., vol. 153, Dekker, New York, 1994, pp. 105-109. MR.1261882 (94m:16028)

[7] L. Fuchs and L. Salce, Modules over valuation domains, Lecture Notes in Pure and Applied Mathematics, vol. 97, Marcel Dekker Inc., New York, 1985. MR786121 (86h:13008)

[8] _ Modules over non-Noetherian domains, Mathematical Surveys and Monographs, vol. 84, American Mathematical Society, Providence, RI, 2001.

[9] P. Griffith, A note on a theorem of Hill, Pacific J. Math. 29 (1969), 279-284. MR0245613 $(39: 6919)$

[10] L. Gruson and C. U. Jensen, Deux applications de la notion de L-dimension, C. R. Acad. Sci. Paris Sér. A-B 282 (1976), no. 1, Aii, A23-A24. MR0401880 (53:5706)

[11] P. Hill, On the decomposition of groups, Canad. J. Math. 21 (1969), 762-768. MR0249507 $(40: 2752)$

[12] T. Y. Lam, Lectures on modules and rings, Graduate Texts in Mathematics, vol. 189, Springer-Verlag, New York, 1999. MR.1653294 (99i:16001)

[13] S. B. Lee, h-divisible modules, Comm. Algebra, 31 (1) (2003), 513-525. MR 1969238 (2004i:13014)

[14] E. Matlis, Divisible modules, Proc. Amer. Math. Soc. 11 (1960), 385-391. MR0116044 $(22: 6839)$

[15] V. S. Ramamurthi and K. M. Rangaswamy, On finitely injective modules, J. Austral. Math. Soc. 16 (1973), 239-248. MR0332882 (48:11207)

[16] L. Salce and P. Vámos, On some classes of divisible modules, Rend. Sem. Mat. Univ. Padova, 115 (2006), 125-136. MR2245591 (2007c:13002)

[17] R. Warfield, Relatively injective modules, unpublished manuscript, 1969.

[18] W. Zimmermann, Rein-injective direkte Summen von Moduln, Comm. Algebra 5 (1977), 1083-1117. MR0450327 (56:8623)

[19] _ On locally pure-injective modules, J. Pure Appl. Algebra 166 (2002), 337-357. MR.1870625 (2002j:16006)

[20] B. Zimmermann-Huisgen, On the abundance of $\aleph_{1}$-separable modules, Abelian groups and noncommutative rings, Contemp. Math., vol. 130, Amer. Math. Soc., Providence, RI, 1992, pp. 167-180. MR.1176118 (93f:16012)

Dipartimento di Matematica Pura e Applicata, Università di Padova, via Trieste 63, I-35121 PAdova, Italy

E-mail address: salce@math.unipd.it 